\title{
Prevalence and antibiotic susceptibility patterns of pseudomonas aeruginosa in urinary tract infections in a Tertiary care hospital, Central Kerala: A retrospective study over 4 years
}

\author{
Regha I R $\mathbf{R}^{1}$ \\ ${ }^{1}$ Dr. Regha I R, Associate Professor, Department of Microbiology, Affiliated to Sree Narayana Institute of Medical \\ Sciences, Chalakka, Ernakulam, Kerala.
}

Corresponding Author: Dr. Regha I R, Associate Professor, Department of Microbiology, Sree Narayana Institute of Medical Sciences, Chalakka, Ernakulam, Kerala. Email id: reghapks@gmail.com

\begin{abstract}
Background and Objective: Pseudomons aeruginosa (P.aeruginosa) is an important uropathogenthat has shown varied antibiotic susceptibility patterns. This study aims to findout the changing trends in theprevalenceand antibiotic susceptibility patterns ofurinary isolates of P.aeruginosa over four consecutive years. Methodology: A retrospective, record based study was conducted on all culture and sensitivity $(\mathrm{C} / \mathrm{S})$ reports ofurine samples obtained in the microbiology lab in a tertiary care centre, Central Kerala (January 2014 -December 2017). The C/S reports which were positive for significant growth of P.aeruginosawere analyzed to findout its prevalence andantibiotic susceptibility patterns. Descriptive statistics was used for data analysisand the results wereexpressed in percentages. Result: Out of total 6622 urine samples received (14\%) showed significant bacteriuria. P.aeruginosa was the third most common uropathogen isolated with an isolation rate of 3.5\%. The antibiotic resistance observedwere Gentamicin (53.1\%), Amikacin (28\%), Cefipime (28\%), Ceftazidime (34.4\%), Ciprofloxacin (43.7\%), Norfloxacin (40.6\%), Ofloxacin (40.6\%), Piperacillin (37.5\%), Piperacilli-Tazobactam (25\%) and Imipenem (28\%). The isolation rates of P.aeruginosa were $3.9 \%, 2.6 \%, 4.5 \%$ and $2.9 \%$ in $2014,2015,2016$ and 2017 respectively and overthe years it maintained its third position.The year wise analysis of antibiotic resistance showed fluctuating pattern except Amikacin,Cefipime and Fluoroquinoles which displayed a decreasingtrend. The reserve drugs like Piperacillin -tazobactam and Imipenem showed alarming drug resistance, although a hopeful reduction in the resistance was noted in 2017. Conclusion: P.aeruginosa remains as a common uropathogen. Drug resistant strains are markedly high in our area. Antibiotic resistance of P.aeruginosado not show a consistent trend over years and vary from region to region. Soeach institution should have an antibiotic policy based on the local antibiogram which is to be renewed regularly.Instead of opting for higher antibioticseach time, strict implementation of restrictive and rotational antibiotic policies and adherence to the concept of "Reserve drugs" should be followed. This is the only modality to inhibit the emergence of resistance strains of all uropathogens especially opportunistic pathogens like P.aeruginosa.
\end{abstract}

Key words: Antibiotic resistance patterns,Pseudomonas aeruginosa, Urinary tract infections.

\section{Introduction}

Urinary tract infections (UTI) are the most common infections encountered by clinicians and one of the leading causes of morbidity in human population [1,2]. Although Escherichia coli. is predominantly associated with the etiology of UTI, other organisms such as Klebsiella pneumonia, Proteus mirabilis, Enterobacter, Citrobacter, Staphy lococcus aureus, Enterococci etc account for most of the rest [1,3]. Aerobic non-

Manuscript received: $4^{\text {th }}$ February 2018

Reviewed: $14^{\text {th }}$ February 2018

Author Corrected: $20^{\text {th }}$ February 2018

Accepted for Publication: 24 $4^{\text {th }}$ February 2018 fermenting gram negative bacilli are now emerging as important uropathogens. Among these non-fermenters, P.aeruginosa is the predominant and most well-known organism $[3,4]$.

P. aeruginosais a ubiquitous, gram-negative bacillus that can survive in myriad of environment such as aquatic and terrestrial [4]. It is a versatile opportunistic pathogen, associated with nosocomial infections along with other serious implications with high rate of morbidity and mortality[5]. According to the report of 
nosocomial infection surveillance system of center for disease control and prevention, $P$. aeruginosa is the third most common organism causing nosocomial urinary tract infections [5].

Treatment of UTI constitutes a great portion of prescription of antibiotics. Urinary pathogenshave shown a changed pattern of susceptibility to antibiotics, showingan increasedresistance to commonly used antibiotics due to extensive and inappropriate use of antimicrobial agents [6,7]. In P.aeruginosa, increasing resistance towards the available antimicrobials preclude the effectiveness of any antimicrobial regimen.

Because of increasing multidrug resistant (MDR) $\mathrm{P}$. aeruginosa isolates in health care settings, infections are difficult to treat, causing life threatening conditions [5].

MDR mechanism in $\mathrm{P}$. aeruginosa are due to lower outer membrane permeability, acquisition of enzymes like $\beta$-lactamases especially extended spectrum enzymes, carbapenemasesoraminogly coside modifying enzymes. Resistance is conferred by the transfer of plasmids which carry genes to produce antimicrobial enzymes [4,5].

Knowledge of the local bacterial etiology and susceptibility patterns are required to trace any change that might have occurred in time so that updated recommendation for optimal empirical therapy of UTI can be made. In this study we focused on P. aeruginosa because,thisbacteria have different therapeutic options when compared to other commonly encountered gram negative uropathogens.

Eventhougha number of studies have been done on the prevalence and antimicrobial resistance patterns of uropathogens, no data have been reported from the present study area on P.aeruginosa causing urinary tract infections.

The aim of the study is therefore to determine changing trends in the prevalence of P.aeruginosa from suspected UTI cases and their antibiotic susceptibilitypatterns to the most commonly used antipseudomonal antibiotics.

\section{Materials and Methods}

A retrospective, record basedstudy was conducted on allculture and sensitivity $(\mathrm{C} / \mathrm{S})$ reports ofurine samples obtained in the microbiology lab at Sree Narayana Institute of Medical Sciences, Ernakulam during four consecutive years (January 2014 -December 2017).

Inclusion criteria: AllUrine $\mathrm{C} / \mathrm{S}$ reports with positive P.aeruginosa showing $\geq 10^{5}$ colony forming units $/ \mathrm{ml}$.

Exclusion criteria: UrineC/S reports of P.aeruginosa from repeat culture of previously recruited patients.

Method: Mid stream urine samples were collected in sterile containers. The samples were cultured on blood agar and CLED agar (Cystine Lactose Electrolyte Deficient) medium with a standard loop and were incubated at $37^{\circ} \mathrm{C}$ overnight.

A growth of $\geq 10^{5}$ colony forming units $/ \mathrm{ml}$ was considered as significant bacteriuria. The P.aeruginosa isolates were identified by conventional biochemical test[8].

Antibiotic sensitivity testing was done by Kirby- Bauer disc diffusion method on Mueller-Hinton agar and interpretations were done according to the Clinical and Laboratory Standard Institute (CLSI) guidelines [9]. Antibiotics against which susceptibility tested were Gentamicin $(10 \mu \mathrm{g})$, Amikacin $(30 \mu \mathrm{g})$, Ciprofloxacin $(5 \mu \mathrm{g})$, Ofloxacin $(5 \mu \mathrm{g})$, Norfloxacin $(10 \mu \mathrm{g})$, Cefepime $(30 \mu \mathrm{g})$, Ceftazidime $(30 \mu \mathrm{g})$, Piperacillin (100 $\mu \mathrm{g})$, Piperazillin -Tazobactam $(100 / 10 \mu g), \quad$ Imipenem $(10 \mu \mathrm{g})$.

Quality control was performed using P.aeruginosa ATCC 27853. The data regarding the number of P.aeruginosa and its susceptibility patterns were collected from the Microbiology lab register.

Statistical analysis: Descriptive statistics was used for analysis. Collected data were entered in MS-Excel and statistical analysis was done using SPSS 15 software and were expressed as percentages.

\section{Result}

Out of 6622 urine samples received in our lab during the study period of 4 years, we got 925 (14\%) culture positive cases with significant bacteriuria. The isolation rate of P.aeruginosa over the four years was 32 (3.5\%). The year wise isolation rate of P.aeruginosais shown in (Table:1).

The antibiotic susceptibility patterns of all P.aeruginosa isolates and year wisedrug resistance pattern ratesare shown in (Table: 2) and (Table:3) respectively. 


\section{Original Research Article}

Table-1: Isolation rates of P.aeruginosa from urine samples.

\begin{tabular}{|c|c|c|c|}
\hline Year & $\begin{array}{c}\text { Total number of } \\
\text { Urine samples }\end{array}$ & $\begin{array}{c}\text { Significant } \\
\text { bacteriuria(\%) }\end{array}$ & Isolation rate ofP.aeruginosa (\%) \\
\hline 2014 & 1353 & $230(17)$ & $9(3.9)$ \\
\hline 2015 & 1659 & $235(14.2)$ & $6(2.6)$ \\
\hline 2016 & 1594 & $222(13.9)$ & $10(4.5)$ \\
\hline 2017 & 2016 & $238(11.8)$ & $7(2.9)$ \\
\hline Total & $\mathbf{6 6 2 2}$ & $\mathbf{9 2 5}(\mathbf{1 4})$ & $\mathbf{3 2}(\mathbf{3 . 5})$ \\
\hline
\end{tabular}

P.aeruginosa was the third most common urinary isolate after E.coli and Klebsiella species during the entire study period and year wise also it maintained its third position. The isolation rate was maximum in 2016.

Table-2: Antibiotic resistance patterns of $P$.aeruginosa isolates $(\mathrm{N}=32)$

\begin{tabular}{|c|c|c|}
\hline Antimicrobial agents & Number & Resistance (\%) \\
\hline Gentamicin & 17 & 28.1 \\
\hline Amikacin & 9 & 28 \\
\hline Cefipime & 9 & 34.4 \\
\hline Ceftazidime & 11 & 43.7 \\
\hline Ciprofloxacin & 14 & 40.6 \\
\hline Norfloxacin & 13 & 40.6 \\
\hline Orfloxacin & 13 & 37.5 \\
\hline Piperacillin & 12 & 25 \\
\hline Piperacillin -tazobactam & 8 & 28 \\
\hline Imipenem & 9 & \\
\hline
\end{tabular}

The drug resistance was highest against Gentamicin followed by Fluoroquinolones.Reserve drugs like Piperacillin tazobactam and Imipenem exhibited similar resistance rates.

Table-3: Year wise antibiotic resistance $(\%)$ of $P$.aeruginosa

\begin{tabular}{|c|c|c|c|c|}
\hline Antimicrobial agents & $\mathbf{2 0 1 4}$ & $\mathbf{2 0 1 5}$ & $\mathbf{2 0 1 6}$ & $\mathbf{2 0 1 7}$ \\
\hline Gentamicin & 44 & 66 & 50 & 57 \\
\hline Amikacin & 22 & 50 & 30 & 14.3 \\
\hline Cefipime & 33 & 50 & 20 & 42.9 \\
\hline Ceftazidime & 44 & 33 & 40 & 14.3 \\
\hline Ciprofloxacin & 44 & 83 & 40 & 14.3 \\
\hline Norfloxacin & 33 & 83 & 40 & 28.6 \\
\hline Orfloxacin & 33 & 83 & 20 & 14.3 \\
\hline Piperacillin & 44.4 & 66 & 20 & 14.3 \\
\hline Piperacillin-tazobactam & 33.3 & 33 & 10 & 20 \\
\hline Imipenem & 44.4 & 50 & & 2 \\
\hline
\end{tabular}

Amikacin, Cefipime and Fluoroquinolones exhibit a decreasing trend over years but Gentamicin, Ceftazidime and Piperacillin shows an increasing pattern of drug resistance. When compared to 2015, Piperacillin- tazobatam and Imipenem shows a tremendous reduction in the drug resistance. 


\section{Original Research Article}

\section{Discussion}

P.aeruginosa has established itself as a significant uropathogen which may cause dreaded complications if not treated properly. Antibiotic resistance is a major clinical problem in treatinginfections caused by this organism. The resistance patterns and isolation rates of P.aeruginosa varies regionally. Hence, increasing importance has been placed on the careful monitoring of antimicrobial resistance patterns of P.aeruginosa isolates for appropriate empirical as well as targeted treatment of the same. The study shows the prevalence and the antibiotic susceptibility patterns of urinary isolates of P.aeruginosa in a tertiary care hospital, Central Kerala.

In the present study P.aeruginosa was the third most common urinary isolate after E,coli and Klebsiella species Otherauthors also observed P.aeruginosa asthe third most frequent urinary isolate [10-13]. In some studies it acquiredsecond positionbut in a recent study from Pakisthan it rankedonly fifth [2,4]. The isolation rates in the present study is compared to some recent studies from India and other countries (Table:4).Eventhough there is a slight variation in the prevalence, P.aeruginosa continue to be an important uropathogen in majorities of the studies.

Table-4 : Prevalence of P.aeruginosa from urine samples in various recent studies

\begin{tabular}{|c|c|}
\hline Various studies & Isolation rateof P.aeruginosa (\%) \\
\hline Present study & 3.5 \\
\hline Bency JAT et al ; 2017; Kerala [10] & 3.8 \\
\hline Singh VP et al; 2017;UP[2] & 6.7 \\
\hline Sangeeta et al; 2017;Maharashtra [13] & 9.85 \\
\hline Shah DA et al ;2015;Karachi [4] & 5.4 \\
\hline Jain et al;2014;Patiala [14] & 9 \\
\hline Prakash D et al; 2013;Meerut [12] & 12.9 \\
\hline Syed MA et al; 2012;Kerala [11] & 2.74 \\
\hline
\end{tabular}

The antibiotic resistanceof P.aeruginosa is compared with various Indian and international studies in (Table:5).

Table-5 : Comparison of Antibiotic resistance patternsof P.aeruginosawith various recent studies

\begin{tabular}{|c|c|c|c|c|c|c|c|c|c|c|}
\hline \multirow[b]{2}{*}{ Various studies } & \multicolumn{10}{|c|}{ Percentage of resistance $(\%)$} \\
\hline & 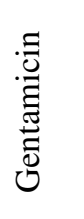 & 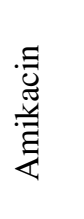 & 苞 & 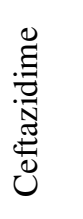 & 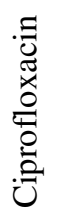 & 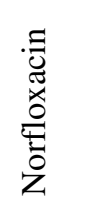 & 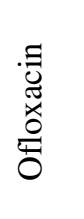 & 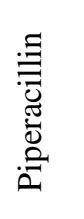 & 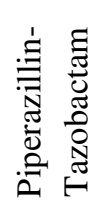 & 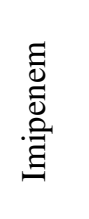 \\
\hline Present study & 53.1 & 28 & 28 & 34.4 & 43.7 & 40.6 & 40.6 & 37.5 & 25 & 28 \\
\hline Singh VP et al[2]. & - & - & - & - & 20 & 14 & - & - & 10 & \\
\hline Shah DA et al[4] & 35.3 & 25.3 & 63.9 & 56.1 & 50 & - & 49 & - & 19.6 & 10.4 \\
\hline BencyJATet al [10] & 30 & 20 & - & 90 & 90 & - & - & 30 & 10 & - \\
\hline Syed MA et al [11] & 49 & 12.5 & 58 & 71 & 21 & 25 & - & - & 0 & 0 \\
\hline Sangeeta et al[13] & 85.7 & 85.7 & - & 100 & - & 42.85 & - & - & 71.4 & 14.28 \\
\hline Oladeinde $\mathrm{BH}$ et al [15]. & 100 & - & - & - & 40 & - & 60 & - & - & - \\
\hline Thomas ss et al [16] & 47 & 47 & 33 & 33 & 60 & - & - & - & 33 & 20 \\
\hline Juayang C et al [17] & 15.8 & 6.7 & - & - & - & - & - & - & 9 & 11.1 \\
\hline
\end{tabular}

In the presentstudy, $53.1 \%$ and $28 \%$ of the isolates were resistant to Gentamicin and Amikacin respectively. Over the 4 years, the resistance to Gentamicin was fluctuating but Amikacin showed a decreasing trend. The Aminoglycosides 


\section{Original Research Article}

inhibit protein synthesis by binding to the $30 \mathrm{~S}$ subunit of the ribosome and the inactivation of the aminoglycosides occurs through the production of enzymes whichtransfer acetyl, phosphate or adenyl groups to the amino acidhydroxyl substituents on the antibiotics [16]. The resistance to Amikacin was highest during the year $2015(50 \%)$, but it was lowerthan resistance to Gentamicin. This shows the growing resistance of P.aeruginosa to Gentamicin. Amikacin has been used sparingly only in severe forms of diseases owing to high cost and the intravenous nature of administration. Therefore, drug resistance has been slow to emerge.In our study, Amikacinis noted to be a comparativelyeffective drug. However, because of itsnumerous side effects including renal toxicity, blurred vision, hearing loss, Bartter-like syndromes neuromuscular blockade, arthralgia, apnoea and many more, it is not commonly used[17].

Cefipime and Ceftazidime are the most frequently prescribed third and fourth generation Cephalosporins respectively [18]. In the present study, Ceftazidime showed $34.4 \%$ and Cefipimedisplayed $28 \%$ of resistance. A recent study from South India showed comparable resistance level but there are some studies showing a very high resistance to both [4,11,16]. In 2017 there was a tremendous decrease in the resistance against Cefipime (14.3\%) but Ceftazidime resistance was almost thrice $(42.9 \%)$ than that of Cefipime. The increased prevalence of Ceftazidime resistant P.aeruginosacan be related to inappropriate use of beta lactam antibiotics.Selective pressure from the use of antimicrobial agents is a major determinant for the emergence of resistant strains[18]. It is recommended to restrict use of Ceftazidime for a period of time to bring the developing resistance under control.

Among Fluoroquinolones, Ciprofloxacin, Norfloxacin and Ofloxacin showed almost similar resistance of $43.7 \%, 40.6 \%$ and $40.6 \%$ respectively. Different authors observed the resistance of ciprofloxacin as low as zero to as high as $90 \%$ $[2,4,10,11,15,16,19]$. Principal modes of Flouroquinolone resistance in P. aeruginosa is due totarget modifications in DNA gyrase (gyr A) andtopoisomerase IV(par C) or mutations in regulatory genesfor efflux pumps that reduce intracellular concentrationsof the antibiotic [20]. Resistance for Fluoroquinolones decreaseddrastically and reached a promising low level of $14.3 \%$ in 2017. Similar study from Punjab also documented a decreasing trend over years [21]. This decreasing trend gives a promising evidenceof rotational antibiotic policy in our institution.

$25 \%$ of the isolates are Pipereacillin -tazobactam resistant. Similar pattern of resistance to Piperacillin- tazobactamwas observed by various authors butan extremely high resistance was noticed by a study conducted by Sangeeta et al $[2,10,13,4,16,17]$. Penicillins are highly ineffective against $\mathrm{P}$ aeruginosa except for Piperacillin-tazobactam because of the beta lactamase inhibitor in addition to the extended spectrum and scare use of the drug [4]. From 2014 to 2017, Piperacillin- tazobactam resistance remained almost consistent with a hopeful dip in 2017.Piperacillin -tazobactam continueto be a good choice of reserve drug for treating UTI caused by P.aeruginosa.

Resistance to Imipenem (28\%) was also noted inthis study. This is very high when compared to other recent studies $[4,13,16,17]$. This increased resistance is quite alarming, taking into account that Carbapenems are the last line of antibiotics for treating Gram-negative bacilli infections. Resistance to Carbapenems may be due to the result of complex interactions of several mechanisms including production of carbapenemase, over production of efflux system and loss of outer membrane porins. P.aeruginosa isolates that are Carbapenem resistant, specifically carbapenemase producing, are the worst, for the reason that they are associated with ahigher mortality rate [17]. When compared to 2015, the resistance rate $(50 \%)$, showed a tremendous decrease in $2017(14.3 \%)$. This may be due to the strict implementation of antibiotic policy and infection control practices in our hospital.

\section{Conclusion}

P.aeruginosa remains as a common uropathogen. Drug resistant strains are markedly high in our area. The susceptibility pattern of one region differs widely from the other. The resistance of P.aeruginosado not have a consistent trend over years. Irregular resistance pattern is observed except in some antibiotics such as Amikacin, Cefipime and Fluoroquinolones which showed a decreasing trend. Reserved drugs like Piperacillin- tazobactam and Imipenem showed an alarming drug resistance. It is emphasized that each institution should have an antibiotic policy based on the antibiogram which should be renewed yearly. Instead of going for higher options of antibioticseach time, strict implementation of restrictive and rotational antibiotic policies and adherence to the concept of 'Reserve drugs" should be followed by each institution.

This is the only modality to inhibit the emergence of resistance strains of all uropathogens especially opportunistic pathogens like P.aeruginosa. 


\section{Original Research Article}

What's New in this study: This study provides information regarding the prevalence and anti microbial susceptibility pattern of urinary isolates of P.aeruginosa.

The study stresses onthe importance of $\mathrm{C} / \mathrm{S}$ reports provided by the microbiology laboratory, so that clinician can select the appropriate antibiotic therapy.

Itemphasizesthe importance of close monitoring of antibiotic susceptibility patterns by preparation of antibiogram and its regular updating.

The study also intends to motivate the strict implementation of restrictive and rotational antibiotic policies and adherence to the concept of reserve drugs.

Funding: Nil, Conflict of interest: None initiated, Permission from IRB: Yes

\section{References}

1. Shylaja TS, Mohankumar. A. Antimicrobial Resistance among Uropathogenic Bacteria in Rural Kerala, India. Int.J.Curr.Microbiol.App.Sci.2017;6(9): 2287-2296.doi.org/10.20546/ijcmas.2017.609.280

2. Singh VP, Mehta A. Bacteriological profile of urinary tract infections at a tertiary care hospital in Western Uttar Pradesh, India. Int J Res Med Sci. 2017 May; 5(5): 2126-9.DOI: http://dx.doi.org/10.18203 / 2320-6012. ijrms 20171855

3. KL S, Rao G G, Kukkamalla A M. Prevalence Of Non-fermenters In Urinary Tract Infections In A Tertiary Care Hospit. Webmed Central MICROBIOLOGY. 2011; 2(1): WMC 001464.doi: 10.9754 / journal. wmc.2011.001464

4. Shah DA, Wasim S, Abdullah FE. Antibiotic resistance pattern of Pseudomonas aeruginosa isolated from urine samples of Urinary Tract Infections patients in Karachi, Pakistan. Pak J Med Sci.2015;31(2):341345. DOI:10. 12669/pjms. 312.6839

5. Trivedi MK, BrantonA,Trivedi D, Nayak, Shettigar $\mathrm{H}$ et al. Antibiogram of Multidrug-Resistant Isolates of Pseudomonas aeruginosa after Biofield Treatment. J Infect Dis Ther.2015;3(5):244. doi:http://dx.doi.org/10. 4172/2332-0877.1000244.

6. Somashekara SC, Deepalaxmi S, Jagannath N, Ramesh B, Laveesh MR, Govindadas D.Retrospective analysis of antibiotic resistance pattern to urinary pathogens in a Tertiary Care Hospital in South India.J
Basic Clin Pharm. 2014 Sep; 5(4): 105-8. doi: 10. 4103/0976-0105.141948.

7. Biswas R, Rabbani R, Ahmed HS, Sarkar MAS, Zafrin N, Rahman MM. Antibiotic sensitivity pattern of urinary tract infection at a tertiary care hospital. Bangladesh Crit Care J. 2014;2(1):21-4.doi: http://dx. doi. org/10. 3329/bccj. v2i1.19952.

8. Collee, J.G., Fraser AG, Marmion BP, Simmons A. Mackey \& McCartney Practical Medical Microbiology. $14^{\text {th }}$ ed. New Delhi, India: Elsevier; 2006.

9. Performance Standards for antimicrobial susceptibility testing; Clinical and laboratory standards institute. Twenty-Third Informational supplement. 2013; 33 (1): M100-S23.

10. Bency JAT, Priyanka R, Jose P. A study on the bacteriological profile of urinary tract infection in adults and their antibiotic sensitivity pattern in a tertiary care hospital in central Kerala, India. Int J Res Med Sci.2017;5(2):666-9. doi: http://dx.doi.org/10.18203/ 2320-6012.ijrms20170171.

11. Syed MA, Ramakrishna PJ, Shaniya K, Arya B , Shakir VP. Urinary Tract Infections - An overview on the Prevalence and the Anti-biogram of Gram Negative Uropathogens in A Tertiary Care Centre in North Kerala, India. J Clin Diag R.2012 September; 6(7): 1192-5.

12. Prakash D, Saxena RS. Distribution and Antimicrobial Susceptibility Pattern of Bacterial Pathogens Causing Urinary Tract Infection in Urban Community of Meerut City, India. ISRN Microbiol. 2013;749629. doi: 10. 1155/ 2013/ 749629.

13. Sangeeta Fattesingh Bhalavi, Vaishali Rahangdale, S.G. Joshi. Study of antibiotic resistance pattern to urinary pathogens in a tertiary care hospital in central India. Int J Sci Res.2017; 6(8): 428-429.

14. Shikha Jain, Geeta Walia, Rubina Malhotra. Prevalence and antimicrobial susceptibility patterns of ESBL producing gram negative bacilli in 200 cases of urinary tract infections. Int J pharm pharm Sci .2014; $6(10): 210-211$.

15. Oladeinde BH, Omoregie R, Olley M, Anunibe JA. Urinary tract infection in a rural community of Nigeria.N Am J Med Sci. 2011 Feb;3(2):75-7. doi: 10.4297/najms.2011.375. 


\section{Original Research Article}

16. Thomas SS, Sreenath K, Sebastian S. Characterization of the antibiotic profile of Pseudomonas aeruginosa isolates from a tertiary care center. Int J Res Med Sci. 2016;4:571-4 DOI: http://dx.doi.org/10. 18203/ 2320-6012.ijrms20160317.

17. Juayang C, Lim T, BonifacioV, Lambot L, Millan SM, Sevilla N, Sy T, Villanueva PJ , Carmina P. Grajales and Gallega $T$. Five-Year Antimicrobial Susceptibility of Pseudomonas aeruginosa from a Local TertiaryHospital in Bacolod City, Philippines. Trop. Med. Infect. Dis. 2017; 2(3): 28 doi: 10.3390/ tropicalmed 2030028 .

18. Mohanasoundaram KM. The Antimicrobial Resistance Pattern in the Clinical Isolates of Pseudomonas Aeruginosa in a Tertiary Care Hospital; 2008-2010 (A 3 Year Study). J Clin Diagn Res.2011 June;5(3):491-494.www.jcdr.net/articles/ PDF/ 1375/ 2267.pdf
19. Olayinka B.O, Olonitola O.S, Olayinka A.T, Agada E.A. Antibiotic susceptibility pattern and multiple antibiotic resistance index of Pseudomonas aeruginosa urine isolates from a University teaching hospital. Afr. J. Clin. Exper. Microbiol.2004 May; 5 (2):198202.

20. Jalal S, Wretlind B. Mechanisms of quinolone resistance in clinical strains of Pseudomonas aeruginosa. Microb Drug Resist. 1998 Winter; 4 (4): 257-61.

21. Sharma N, Gupta AK, Walia G, Bakhshi R. A retrospective study of antimicrobial resistance pattern of Pseudomonas aeruginosa isolates from urine samples over last three years (2013-2015). Int J Basic Clin Pharmacol. 2016 ;5(4):1551-4. DOI: http://dx.doi.org/ 10. 18203/ 2319-2003.ijbcp20162470.

\section{How to cite this article?}

Regha I R. Prevalence and antibiotic susceptibility patterns of pseudomonas aeruginosa in urinary tract infections in a Tertiary care hospital, Central Kerala: A retrospective study over 4 years. Trop J Path Micro 2018;4(1):52-58.doi: 10.17511/jopm.2018.i1.09. 